\title{
La enseñanza para la comprensión y los conceptos estructurantes: una estrategia para el desarrollo de los niveles de comprensión de los estudiantes.
}

Por: Morales, Diana P.1

El diseñar, implementar y evaluar una estrategia de enseñanza basada en la enseñanza para la comprensión (EpC) y los conceptos estructurantes suscita la reflexión sobre la enseñanza de las ciencias, sobre la finalidad que se plantea actualmente con respecto a la formación de los estudiantes: si es la acumulación de conocimientos o su desarrollo cognitivo; de igual forma, surgen interrogantes en relación al planteamiento de proyectos curriculares, donde no bastaría el establecer los contenidos de enseñanza, sino que sería necesario trascender a la formulación de objetivos y metas próximas a las necesidades y características de los estudiantes.

En ese orden de ideas, la EpC desde sus componentes (tópicos generativos, metas de comprensión, desempeños de comprensión y la evaluación diagnóstica continua) propicia la planificación del proceso de enseñanzaaprendizaje, incluyendo no sólo la organización los conceptos de enseñanza, sino la formulación de los objetivos e indicadores del mismo. Asimismo, el reconocimiento de los conceptos que favorecen el desarrollo cognitivo de los educandos permite evitar la saturación de los proyectos curriculares con contenidos aislados.

\section{SITUACIÓN PROBLEMA}

Desde las prácticas y acercamientos que han realizado algunos estudiantes del Departamento de Biología, de la Universidad Pedagógica Nacional, a las escuelas se reconoce que con frecuencia se plantea que una de las mayores dificultades es que los estudiantes memorizan los contenidos que el docente les expone y luego de aprobar su evaluación los olvidan. Partiendo de la anterior afirmación, surge un interrogante ¿durante las clases de ciencias, se está favoreciendo la memorización de conceptos o su comprensión? Sin embargo, la respuesta a éste se ve influida por la idea que el maestro tiene de la enseñanza, y a lo que se entiende por comprensión. A la par, permanentemente los docentes se preguntan qué contenidos es importante enseñar y cuáles a portan en mayor medida a los alumnos.

Estudiante de octavo semestre de Licenciatura en Biología. Universidad gógica Nacional, 2009. patricia dianis@hotmail.com 


\section{LOS CONCEPTOS ESTRUCTURANTES}

Cuando en un proyecto curricular no se tiene como principal objetivo el que los estudiantes memoricen una serie de términos, sino que logren comprender las temáticas que se les presentan y el desarrollo actitudes, es pertinente reflexionar sobre la forma en que se establecen los contenidos de enseñanza. En la enseñanza de las ciencias es común encontrar programas saturados por conceptos y en consecuencia los alumnos no logran comprender las temáticas, por lo cual, éstas son olvidadas con gran facilidad. En ese sentido, se plantea diseñar e implementar una propuesta donde se establezcan los conceptos que realmente contribuyen al proceso de aprendizaje de los educandos, a demás, de reconocer las relaciones que se puedan establecer entre los mismos. Lo anterior ha sido propuesto por diversos autores, entre ellos Armúa de Reyes (2006), Astolfi (1994, en MERINO, G.) y Gagliardi (1986 y 2008), recibiendo el nombre de conceptos estructurantes.

En la propuesta que realiza cada uno de los autores mencionados anteriormente no se encuentran mayores diferencias, por el contrario comparten objetivos como el promover una visión integral de las ciencias y presentar a los estudiantes contenidos que permitan su desarrollo cognitivo. Armúa de Reyes (2003, en Armúa de Reyes, 2006), considera que "trabajar con conceptos estructurantes introduce diferencias en la formas habituales de seleccionar contenidos escolares que se centran en el dato o fenómeno aislado, para dar lugar a propuestas didácticas globalizadoras e integradoras". Planteamiento desde el cual cobra importancia el realizar la contextualización de una institución, con el fin de promover un proceso educativo contextual y en consecuencia que contribuya a las necesidades de esa población en particular. Con una orientación similar, Astolfi (1994) define los conceptos estructurantes como "un conjunto de tramas generales de conocimientos que integran elementos de diversas disciplinas y del propio contexto sociocultural".

Por su parte, Gagliardi (2008) plantea que cuando un estudiante aprende un concepto estructurante, logra cambios en su capacidad de aprendizaje, y en esa medida le "es posible incorporar nueva información y forjar nuevos conocimientos; simultáneamente se desarrolla una nueva capacidad para observar el mundo". En consecuencia, este autor considera que "la instrucción debe estar orientada a enseñar un cierto número de conceptos que- una vez interiorizados- transformen el sistema cognitivo y conduzcan a una nueva estructura conceptual que permita avanzar en el aprendizaje". Además, Gagliardi (1986), considera que la transformación del sistema cognitivo permite comprender los nuevos conocimientos y reorganizar y/o transformar los anteriores. Pozo (1999) plantea que el cambio conceptual implica no solo la modificación de los conceptos, sino que a su vez se deben presentar cambios en los procesos y representaciones por medio de las cuales los alumnos comprenden los fenómenos científicos. 
Partiendo de lo anterior, se propone diseñar e implementar una estrategia educativa basada en conceptos estructurantes, por medio de los cuales se logre interesar a los estudiantes, teniendo en cuenta que, durante la selección de contenidos se deben involucrar elementos contextuales, de tal forma que, los educandos logren establecer relaciones entre los nuevos conceptos y su conocimiento cotidiano y en la medida de lo posible una mejor comprensión de los contenidos de enseñanza.

\section{LA ENSEÑANZA PARA LA COMPRENSIÓN}

En primer lugar, según Gardner (2000) “cuando un estudiante comprende- un concepto, una temática, una técnica, una teoría o un ámbito de conocimiento-, lo puede aplicar de una forma apropiada en una nueva situación". Con el fin de motivar dicha comprensión, el autor propone tres estrategias; la primera hace alusión a la importancia de la interacción estudiante-maestro, por medio de experiencias prácticas; la segunda, consiste en presentar a los alumnos situaciones de su vida cotidiana, con el objetivo de que logren comprobar o refutar su conocimiento ellos mismos; un marco de referencia facilita la comprensión por parte de los estudiantes, ello quiere decir, que es oportuno crear espacios donde los estudiantes puedan aplicar sus conocimientos.

Otro de los autores que ha trabajado alrededor de la comprensión es Perkins (en Stone, 1999), quien considera que “...comprender es la habilidad de pensar y actuar con flexibilidad a partir de lo que uno sabe. (...) la comprensión de un tópico es la capacidad de un desempeño flexible". En ese sentido, tener un desempeño flexible involucra ir más allá del conocimiento (memorización) y la habilidad, es decir, se requiere explicar, justificar, extrapolar y aplicar un tópico en diferentes situaciones.

Gardner (2000) y Stone (1999) plantean cuatro elementos fundamentales en la EpC: tópicos generativos, metas y desempeños de comprensión y evaluación continua. Stone (1999) afirma:

"cada elemento contribuye (...)identificando tópicos o temas generativos y organizando propuestas curriculares alrededor de ellas; clarifican lo que los estudiantes deben comprender articulando metas claras y centradas en comprensiones claves; motiva el aprendizaje de los alumnos involucrados en desempeños de comprensión que exigen que éstos apliquen, amplíen y sinteticen lo que saben, y controla y promueve el avance de los estudiantes por medio de evaluaciones diagnósticas continuas de sus desempeños, con criterios directamente vinculados con las metas de comprensión"

\section{RELACIÓN EpC- CONCEPTOS ESTRUCTURANTES}

Desde lo expuesto hasta el momento, con respecto a la $\mathrm{EpC}$, se reconocen algunos elementos que permiten pensar en el diseño de una unidad de enseñanza centrada en 
este modelo y los conceptos estructurantes, entre ellos los tópicos generativos, teniendo en cuenta las relaciones que se pueden establecer a través de ellos; asimismo, se hace evidente que el objetivo en cada una de estas estrategias no es la acumulación de conocimientos sino la comprensión de determinadas temáticas, las cuales, dependiendo la forma en que se orienten, pueden propiciar el desarrollo del sistema cognitivo de los estudiantes.

\section{¿EXISTEN NIVELES DE COMPRENSIÓN?}

Alrededor del tema se encuentran los trabajos de Benjamín Bloom (1975) y Boix y Gardner (en Stone, 1999). Desde la revisión realizada, se considera que la propuesta del primero se incluye de una u otra forma en los planteamientos de éstos últimos. Esta afirmación se realiza teniendo en cuenta que los puntos clave de los tres niveles propuestos por Bloom se pueden encontrar dentro de las cuatro dimensiones y cuatro niveles de comprensión que se enmarcan en la EpC (propuesta de Boix y Gardner, en Stone, 1999).

Benjamín Bloom (1975) propone tres niveles de comprensión: la traducción, la interpretación y la extrapolación. El primero, hace referencia a los estudiantes que logran expresar un texto en otras palabras o lo pasan de un símbolo a otro; el segundo nivel, se hace evidente cuando los alumnos más allá del reconocimiento de las partes separadas de una comunicación pueden descubrir las interacciones entre las partes y relacionarlas con hechos reales, además, reconoce los elementos esenciales de la temática; y el nivel de extrapolación exige que los estudiantes realicen inferencias y propongan algunas conclusiones o posibles consecuencias respecto a la unidad trabajada.

Por su parte, Boix y Gardner (en Stone, 1999) plantean cuatro dimensiones y cuatro niveles de comprensión, dentro de los cuales se pueden describir los desempeños de los alumnos. Es de aclarar que, dentro de cada dimensión puede desarrollarse un nivel diferente de comprensión, es decir, un estudiante puede presentar un nivel de aprendiz en la dimensión de contenidos, pero su nivel en la dimensión de propósitos es de novato.

- La primera dimensión es la de contenido, por medio de ésta se evalúa en qué nivel trascienden las perspectivas intuitivas de los estudiantes, así como "el grado hasta el cual pueden moverse con flexibilidad entre ejemplos y generalizaciones en una red conceptual coherente y rica" (Boix y Gardner, en Stone, 1999).

- La segunda dimensión, corresponde a los métodos, en ella se evalúa la capacidad de los estudiantes de cuestionarse sobre lo que conocen y lo que se les dice, al igual que, el uso de métodos confiables para construir y validar sus afirmaciones. 
- En la dimensión de propósitos se evalúa la capacidad de los estudiantes para establecer los propósitos que orientan la construcción del conocimiento y su capacidad para emplear éste en múltiples situaciones, así como el que reconozcan las consecuencias de hacerlo.

- En la cuarta y última dimensión, formas de comunicación, se valora el uso de sistemas de símbolos para expresar lo que saben dentro de los tipos de desempeños establecidos.

Dentro de las dimensiones descritas anteriormente los educandos pueden presentar cuatro niveles de profundidad: desempeños de comprensión ingenua, novatos, aprendiz y desempeños de comprensión de maestría.

- Desde los primeros, los estudiantes basan sus explicaciones en el conocimiento intuitivo, en ese sentido, no establecen relaciones entre lo que aprenden en la escuela y su conocimiento cotidiano.

- A partir de los segundos, los alumnos inician exponiendo algunos conceptos disciplinares y establecen relaciones simples entre ellos, de igual forma, consideran los métodos de la disciplinas como una receta.

- En los desempeños de comprensión de aprendiz, demuestran el uso flexible de conceptos de la disciplina, si se les orienta, los desempeños de este nivel vislumbran la relación entre el conocimiento disciplinar y el cotidiano.

- Desde los desempeños de comprensión de maestría, predominan los pensamientos integradores, creativos y críticos, por ello, "los alumnos pueden usar el conocimiento para reinterpretar y actuar en el mundo que los rodea." (Boix y Gardner, en Stone, 1999).

\section{CONCLUSIONES}

Es necesario reflexionar respecto a los objetivos que se están planteando en la enseñanza de las ciencias, con el fin de evaluar y mejorar las estrategias que se emplean en el proceso educativo. A la par, debe reconocerse la importancia del contexto de los estudiantes, así como de su conocimiento cotidiano en la construcción del conocimiento escolar.

La enseñanza para la comprensión y los conceptos estructurantes son dos estrategias de enseñanza que orientan la planificación, ejecución y evaluación del proceso de enseñanza- aprendizaje; en éstas se tiene en cuanta tanto a los estudiantes como al docente. De igual forma, desde éstas se promueve el fortalecimiento de los niveles de comprensión que desarrollan los estudiantes, trascendiendo de la memorización a la argumentación y explicación de diversas situaciones, que en determinado momento pueden estar dentro de su vida cotidiana. 


\section{Bibliografía}

- ARMÚA, M. 2006. Aplicación de metaconceptos en la enseñanza de las ciencias naturales. Memorias tercer Encuentro de Investigadores en Didáctica de la Biología.

- ASTOLFI, J. 1994. Los obstáculos para el aprendizaje de conceptos en ciencias: la forma de franquearlos didácticamente. En MERINO, G. enseñar ciencias naturales en el tercer ciclo de la E.G.B. Madrid/Buenos Aires: Editorial Aíque.

- BLOOM, B. 1975. Evaluación del aprendizaje. Vol. 1. Ediciones Troquel: Buenos Aires. 419 p.

- GAGLIARDI, Raúl. 2008. Enseñar Biología: para qué, cómo, cuándo? Algunos elementos de la didáctica de la Biología. Memorias, $V$ Encuentro Nacional de Experiencias en Enseñanza de la Biología y la Educación Ambiental. Colombia.

- GAGLIARDI, Raúl. 1986. Los conceptos estructurantes en el aprendizaje por investigación. Enseñanza de las ciencias. 4 (1): 30-35.

- GARDNER, H. 2000. La educación de la mente y el conocimiento de las disciplinas. Lo que todos los estudiantes deberían comprender. Paidos: Barcelona. 409 p.

- POZO, J. 1999. Más allá del cambio conceptual: El aprendizaje de la ciencia como cambio representacional. Enseñanza de las ciencias. 17 (3): 513-520.

- STONE, M. 1999. La enseñanza para la comprensión. Vinculación entre la investigación y la práctica. Paidos: Barcelona. 396 p. 